\title{
Analysis of systemic epigenetic alterations in inflammatory bowel disease: defining geographical, genetic, and immune-inflammatory influences on the circulating methylome
}

Rahul Kalla ( $\boldsymbol{\nabla}$ Rahul.kalla@ed.ac.uk)

University of Edinburgh https://orcid.org/0000-0002-3276-6751

Alex Adams

University of Oxford

Jan Nowak

Poznan University of Medical Sciences

Daniel Bergemalm

Örebro University

\section{Simen Vatn}

Akershus University Hospital https://orcid.org/0000-0002-6124-4995

Nicholas Ventham

University of Edinburgh

Nicholas Kennedy

University of Exeter

\section{Petr Ricanek}

Akershus University Hospital

Jonas Lindstrom

University of Oslo

IBD Character Consortium

University of Edinburgh

Johan Söderholm

Linköping University https://orcid.org/0000-0002-3250-5367

Marieke Pierik

Maastricht University Medical Centre

Mauro D\&\#39;Amato

CIC bioGUNE https://orcid.org/0000-0003-2743-5197

Fernando Gomollon

Hospital Clínico Universitario Lozano Blesa

Christine Olbjørn

Akershus University Hospital https://orcid.org/0000-0001-5680-4313 


\section{Rebecca Richmond}

University of Bristol https://orcid.org/0000-0003-0574-5071

\section{Caroline Relton}

University of Bristol

\section{Jorgen Jahnsen}

University of Oslo

\section{Morten Vatn}

University of Oslo

Jonas Halfvarson

Orebro University

Jack Satsangi

University of Oxford https://orcid.org/0000-0002-6357-9684

\section{Article}

Keywords: DNA methylation, genetics, inflammatory bowel diseases (IBD), prognosis, methylation quantitative trait loci, gene expression, epigenetic clock, mendelian randomisation

Posted Date: May 27th, 2021

DOl: https://doi.org/10.21203/rs.3.rs-537439/v1

License: (c) (i) This work is licensed under a Creative Commons Attribution 4.0 International License. Read Full License

Version of Record: A version of this preprint was published at Journal of Crohn's and Colitis on August 24th, 2022. See the published version at https://doi.org/10.1093/ecco-jcc/jjac127. 


\section{Abstract}

Gene-environment interactions play a pivotal role in the pathogenesis of Inflammatory Bowel Disease (IBD). Using the IBD Character inception cohort (295 controls, 154 CD, 161 UC, 28 IBD-U) we confirm the presence of a highly characteristic DNA methylation profile in peripheral blood implicating 137 differentially methylated positions (DMP) in IBD, including VMP1/MIR21 $\left(\mathrm{p}=9.11 \times 10^{-15}\right)$ and RPS6KA2 $\left(6.43 \times 10^{-13}\right)$; with consistency seen across Scandinavia and UK. Cell of origin analysis preferentially implicated the monocyte lineage. Dysregulated loci demonstrate strong genetic influence, notably VMP1 $\left(p=1.53 \times 10^{-15}\right)$. Age acceleration is seen in IBD (coefficient $0.94, p<2.2 \times 10^{-16}$ ). Several immuno-active genes demonstrated highly significant correlations between methylation and gene expression in IBD, in particular OSM: IBD r - 0.32 , p $3.64 \times 10^{-7}$ vs. non-IBD r $-0.14, p=0.77$ ). Multi-omic integration of methylome, genome and transcriptome also implicate specific pathways that associate with immune activation, response and regulation at disease inception. At follow up, a signature of 3 DMPs (TAP1, TESPA1, RPTOR) associated with treatment escalation to biological agents or surgery (hazard ratio of $5.19\left(\mathrm{Cl}: 2.14-12.56\right.$, logrank $\left.\mathrm{p}=9.70 \times 10^{-4}\right)$.

\section{Introduction}

Inflammatory Bowel Diseases (IBD) phenotypically classified in two main entities, Crohn's disease (CD) and Ulcerative Colitis (UC) represent an important public health concern, with an projected prevalence of $1 \%$ in Western populations by 2030 . There are significant implications for healthcare planning as costs, particularly of new treatments, increase ${ }^{1}$. These considerations have accelerated global efforts in better understanding the aetiology of IBD. Unequivocal data now implicate the interaction of host susceptibility with the exposome in the development of IBD ${ }^{2}$. These data have inevitably stimulated studies to explore the potential importance of epigenetic mechanisms, including DNA methylation, in pathogenesis. DNA methylation may regulate gene expression through its effect on the chromatin state as well as the accessibility of the transcription binding $\operatorname{sites}^{3-5}$ and can be influenced by many pertinent environmental factors including smoking and age $e^{6,7}$.

We have previously identified several alterations in the IBD-associated circulating epigenome. Our initial study in the high-prevalence Scottish population characterised a replicable pattern of DNA alterations in children with $C D$, with highly significant enrichment of methylation changes around GWAS single nucleotide polymorphisms, in particular the HLA region and VMP1/MIR2 $1^{8}$. More recently, our adult epigenome-wide study within the Scottish population identified distinct differential methylation across key IBD genes ${ }^{9}$. Certain signals were highly cell-specific; RPS6KA2 was shown to be a CD $14^{+}$monocyte specific signal in $\mathrm{IBD}^{9}$. These top differentially expressed methylation signals were independently confirmed and replicated in a treatment-naïve paediatric CD cohort in North America (RISK consortium) ${ }^{10}$, providing strong stimulus for further research in this field. Importantly, the extent to which the findings can be generalised to other populations is largely unknown. Furthermore, the timing, stability, and 
functional importance of epigenetic alterations in affecting gene transcription have not been fully investigated.

With the advancing therapeutic repertoire in IBD, there is keen interest in risk stratifying patients at diagnosis in order to allow personalised medicine, with recent optimism that genetic, transcriptional, glycomic or serological markers may predict disease course, and help position new therapies ${ }^{11-18}$. Promising data have emerged that epigenetic alterations are helpful in other diseases, notably colorectal cancer. The potential clinical utility of these epigenetic marks as biomarkers in immune- mediated diseases is yet to be determined.

In our multi-centre study, we aim to extend current understanding of the pathogenetic and translational importance of the circulating epigenome in IBD. We firstly aim to assess the consistency of DNA methylation alterations in IBD across geographically distinct populations in UK, Scandinavia and Spain. We investigate environmental modifiers of the methylome including inflammation, smoking and aging. Furthermore, we define the genetic contribution to these alterations, their association with gene expression and define the methylome of disease progression in IBD. Whilst focussed on IBD, the findings have potential implications beyond this disease field, to other complex and immune-mediated diseases.

\section{Results}

\section{Study demographics}

The IBD Character cohort represents a multi-centre inception cohort in which 247 (72\%) of the 343 IBD patients were treatment naïve at recruitment. Genome-wide methylation was measured in 638 DNA samples extracted from peripheral blood (295 controls, 154 CD, 161 UC and 28 IBD-unclassified (IBD-U) patients). Table 1 summarises study recruitment and patient demographics. The mean age in patients with IBD ( $n=343)$ was 34 years (range $7-79)$, and 33 years (range 3-79) in controls $(n=295)$. A total of $27 \%$ of CD patients had a colonic disease phenotype at recruitment while $42 \%$ of patients with UC exhibited extensive colitis. Two hundred individuals were recruited in the UK, 367 in Scandinavia and 66 in Spain at presentation for investigation of suspected IBD. A total of 33\% of those recruited in the UK had confirmed IBD after investigation, while 58\% and 39\% had IBD in the Scandinavian and Spanish cohort respectively (Table 1 ).

\section{Differentially methylated probes in Inflammatory Bowel Disease}

Across the entire cohort, 137 probes exhibited Holm significant IBD-associated methylation differences in comparing IBD with controls (Supplementary Table 1). These include probes mapping at the loci VMP1/MIR21 $\left(\mathrm{p}=9.11 \times 10^{-15}\right), \operatorname{SBNO2}\left(2.70 \times 10^{-14}\right), \operatorname{RPS} 6$ KA2 $\left(6.43 \times 10^{-13}\right)$, and TNFSF10 $\left(7.72 \times 10^{-8}\right)$, thereby replicating and validating our previous findings ${ }^{9}$. Novel findings include differential methylation

of PHOSPHO1 $\left(3.43 \times 10^{-9}\right)$ and SELPLG $\left(2.54 \times 10^{-7}\right)$. Table 2 summarises the top differentially methylated positions (DMPs). 
Similar analyses were performed to identify UC and CD specific DMPs. There were 72 DMPs that differentiated CD from controls (Supplementary Table 2) and 67 DMPs that differentiated UC from controls (Supplementary Table 3). There were 24 DMPs that demonstrated overlap across UC and CD analyses (Supplementary Figure 1). There were no probes that differentiated UC from CD. Analysis was performed of Differentially Methylated Regions (DMRs), defined as regions with $\geq 3$ contiguous probes with FDR corrected $p<0.05$ within a distance threshold based on expected probe density. The VMP1 locus on chromosome 17 was the only DMR identified within our dataset that remained significant using these criteria.

Next, we compared our dataset with the previous findings from our group on DMPs in adult and paediatric IBD by correlation analyses of the top 1000 methylation probe beta values in the 3 cohorts (Supplementary Figure 2) ) $^{8,9}$. Strong correlation was seen across populations (adult BIOM cohort ${ }^{9} \mathrm{r} 0.96$, $\mathrm{p}<2.20 \times 10^{-308}$; paediatric cohort $\mathrm{r} 0.81, \mathrm{p}<2.20 \times 10^{-308}$ ).

\section{Consistency of Differentially methylated positions (DMPs) across Northern Europe}

We then analysed the consistency of methylation across Europe (Figure 1) by splitting our cohort based on geographic area (Scandinavia vs UK vs Spain). Independent DMP analysis using all probes was performed in Scandinavia, identifying 34 probes that differentiated IBD from controls with a Holm $p<0.05$ (Table 3). These included SBNO2 $\left(p=2.88 \times 10^{-9}\right), \operatorname{VMP} 1\left(\mathrm{p}=6.89 \times 10^{-8}\right)$ and RPS6KA2 $\left(\mathrm{p}=1.57 \times 10^{-3}\right) . \mathrm{A}$ total of 26 of these probes were significant in the UK cohort $(n=200)$. In the Spanish cohort $(n=66)$ only one (RPS6KA2) remained significant ( $\mathrm{Holm} \mathrm{p}=0.03$ ). Power calculations were performed in view of these findings, taking into consideration the effect sizes noted in the Scandinavian cohort. This post-hoc analysis confirmed that the Spanish cohort was adequately powered to detect significant differences in 11 of the 34 DMPs identified in the Scandinavian dataset (power $=0.8$, alpha $=0.05$, effect size cut-off $=0.8$; Supplementary Table 4). DMPs consistently replicated across samples from UK and Scandinavia notably $Z E B 2, S B N O 2$ and ZPLD1 were not detected in samples from Spain despite being adequately powered to detect these DMPs.

\section{Deciphering tissue and immune cell specificity of differentially methylated probes in IBD}

Blood cell-type-specific DNase hypersensitive sites (DHS) enrichment testing using the eFORGE v2.0 online tool ${ }^{19}$ was performed for our top 137 differentially expressed methylation probes. This demonstrated that 62 of the 137 IBD associated CpGs are enriched in DHSs within monocytes (binomial $\left.\mathrm{p}=2.04 \times 10^{-8}\right)$. These encompassed the top-most differentially expressed DMPs in disease such as OSM, VMP1, SELPLG (PSGL-1) and AIM2. No enrichments were seen in other immune cells such as T-cells, Bcells, NK cells or haematopoietic stem cells (Supplementary Figure 3).

We then examined for overlap between the circulating blood DMPs in CD and UC in this study and those differentially methylated in human colonic intraepithelial cells (IECs) in CD and UC cohorts ${ }^{20}$. A total of 38 and 5 probes overlapped in UC and CD respectively (Supplementary table 5). Top IEC signals with 
overlap in circulating methylome across both UC and colonic CD included SBNO2, RPS6KA2 and SELPLG. Overlapping probes unique to UC included AIM2, MAD1L1, MIR4470 and MIR3679.

\section{The association of DNA methylation with inflammation}

In order to better understand the influence of inflammation on the top differentially methylated probes, 3 distinct analyses were performed. These included correlations with inflammatory markers, DMP analysis using inflammatory markers such as hsCRP as covariates and DMP overlap with published inflammation associated methylation probes.

We investigated the correlation of the 137 differentially expressed DMPs in IBD with inflammatory markers, i.e. high sensitivity c-reactive protein (hsCRP) and albumin, in individuals with complete data $(n=591$; Supplementary Figure 4). A total of 98 probes correlated with concentration of hsCRP and 118 with albumin levels. Most significant correlations with hsCRP included LIPC (cg27307975, r -0.56, p= $\left.<2.22 \times 10^{-16}\right)$ and ZEB2 (cg20995564, $\left.\mathrm{r}-0.48, \mathrm{p}=<2.22 \times 10^{-16}\right)$. Top DMPs such as VMP1 (cg12054453, $\mathrm{r}$ $\left.0.25, \mathrm{p}=3.78 \times 10^{-8}\right)$ and RPS6KA2 (cg17501210, $\left.\mathrm{r}-0.30, \mathrm{p}=7.81 \times 10^{-12}\right)$ showed moderate correlation with hsCRP. There were however 39 probes that did not correlate with hsCRP and included another VMP1 probe (cg02782634, $r-0.13, p=0.08$ ) (Supplementary Table 6). In order to adjust for inflammatory activity, we also performed DMP analysis with previously described covariates and with the addition of hsCRP. A total of 59 probes remained significant and included SBNO2 $\left(\mathrm{p}=5.26 \times 10^{-14}\right), \operatorname{RPS} 6 K A 2\left(\mathrm{p}=4.51 \times 10^{-11}\right)$ and VMP1 $\left(p=5.36 \times 10^{-10}\right)($ Supplementary Table 7$)$.

We then compared our top DMPs to those that have been reported to be associated with CRP levels ${ }^{21}$. A total of 43 of the 218 DMPs identified in the Lighthart et a/ were differentially methylated in our study. There were however 94 probes that still associated with IBD and were independent of inflammation associated probes (Supplementary Table 8). These included CLU(cg16292768), SBNO2 (cg12170787) and VMP1(cg02782634).

Finally, in order to identify inflammation independent DMPs, we excluded probes that correlated with hsCRP or reported inflammation-associated DMPs and identified 30 DMPs that showed no overlap with published inflammation associated DMPs or any correlation with inflammation (Supplementary Table 9).

\section{The association of DNA methylation with smoking}

Smoking can affect DNA methylation at certain CpG sites in blood and a recent systematic review summarised a total of 1460 smoking-associated CpG sites ${ }^{22}$. None of the 137 probes identified in this study overlap with the reported smoking-associated $\mathrm{CpGs}$. Given the divergent effects of smoking on IBD subtypes, we performed analyses for probes that associate with CD or UC by including smoking, age, sex and cell proportions as covariates. In CD, 107 DMPs remained significant; SBNO2, RPS6KA2 and VMP1 being the top probes (Supplementary Table 10). In UC, 59 probes remained significant: SBNO2, VMP1 and ZEB2 being the most significant probes (Supplementary Table 11). 


\section{'Epigenetic age' acceleration and its association with Inflammatory Bowel Disease}

The 'epigenetic age' of the patients was calculated using the methodology described by Horvath et aP . There was a strong correlation between actual age and 'epigenetic age' in this cohort ( $r=0.94, \mathrm{Cl}$ : 0.930.95, p<2.20 $\times 10^{-16}$; Supplementary Figure 5). Age acceleration (AgeAcce) was seen in IBD (coefficient $\left.0.94, p<2.2 \times 10^{-16}\right)$. DiffAge, defined as the difference between predicted biological age and chronological age was determined for IBD and controls (including subtypes) ${ }^{7,23}$. There were significant DiffAge seen between IBD and controls (non-IBD: median 4.34 years (IQR:3.83-4.70) vs. IBD: 5.28 years(IQR: 4.72-5.64); $\mathrm{p}<2.20 \times 10^{-16}$ ). Differences were also seen between IBD subtypes compared to non-IBD (vs. UC: 5.08 years (IQR: 4.51-5.49); $\mathrm{p}<2.20 \times 10^{-16}$ and vs. CD: 5.53 years (IQR: 4.99-5.79); $\mathrm{p}<2.20 \times 10^{-16}$; Supplementary Figure 5). There was poor correlation seen between DiffAge and inflammatory markers in the entire cohort (hsCRP: $r$ 0.09, $p=0.03$; Alb: $r-0.13, p=2.88 \times 10^{-3}$ ). No correlations were seen between DiffAge and treatment exposure in $U C$ or $C D(U C p=0.89 ; C D p=0.21)$.

\section{Germline variations show a strong correlation with DNA methylation (meQTLs)}

Using paired genetic and methylation data for the entire cohort $(n=638)$ and age, sex as covariates, meQTLs were generated using the top DMPs using the entire cohort (DMPs $n=137$ ). A total of 2991 cismeQTLs were identified. After applying a MAF $>0.05$ and holm adjustment, 341 cis-meQTLs remained significant across 21 unique genes, indicating a strong genetic influence on methylation. Several key loci that were significantly differentially methylated in IBD had a strong genetic influence including ITGB2 (7 cis-meQTLs; top $\mathrm{p}=2.83 \times 10^{-16}$ ), and $143 \mathrm{VMP} 1 /$ MIR21 cis-meQTLs across 6 probes (Supplementary Table 12). This includes meQTLs with a known GWAS single nucleotide polymorphism (snp) rs 1292053 and also with its previously reported LD snp ( $r s 8078424, r^{2}=0.43$, top $\left.p=1.48 \times 10^{-20}\right)^{9}$. Other novel IBD relevant associations include $A / M 2\left(16\right.$ cis-meQTLs; top $\left.\mathrm{p}=2.83 \times 10^{-16}\right)$.

In order to determine the causal role of DNA methylation in IBD, Mendelian randomisation was applied to our dataset using TwoSampleMR ${ }^{24}$. The most significant meQTLs for each CpG (sentinel meQTL) were generated using all snps and methylation probes independent of a diagnosis of IBD (Supplementary Table 13). Using sentinel meQTLs as the instrument variable, methylation as exposure variable and IBD as outcome variable, no causal associations were identified in our dataset.

\section{IBD associated genes are differentially methylated and correlate with gene expression}

In this study, paired whole blood gene expression data was available in 590 patients. Of the 137 DMPs identified, 24 probes were located either 200 or 1500 nucleotides upstream of the transcriptional start sites (TSS200 and TSS1500), within regulatory regions of a gene (5'untranslated region ( $5^{\prime}$ UTR), 3' untranslated region (3'UTR)) or body of the gene. In this study, we discovered 81 highly significant correlations (Supplementary Table 14) with a series of novel genes demonstrating high correlation between methylation alterations and expression including $C D 247$ (Body, $r=-0.69, \mathrm{p}=4.70 \times 10^{-80}$ ), SELPLG ( $\left.5^{\prime} U T R, r=-0.56, p=3.10 \times 10^{-46}\right), O S M\left(T S S 1500, r=-0.33, p=2.48 \times 10^{-14}\right)$ and AIM2 (TSS1500, $r=-0.51$, 
$\left.\mathrm{p}=6.32 \times 10^{-37}\right)$. Further sub-analysis was performed investigating the correlation differences in IBD and non-IBD cohorts (Supplementary Table 15). SELPLG expression and methylation demonstrated a relative higher negative correlation within IBD than non-IBD (IBD r $-0.53, p=2.24 \times 10^{-22}$ vs $-0.37, p=6.34 \times 10^{-8}$; $\left.5^{\prime} U T R\right)$. A total of 14 probes demonstrated significant correlation in IBD patients but no correlation in nonIBD (Figure 2). Examples include ZEB2 demonstrated negative correlation in IBD $\left(r-0.45, p=6.43 \times 10^{-15}\right.$; gene body) and no significant correlation in controls $(r-0.16, p=0.29)$. Similarly, OSM expression negative correlated with methylation within IBD cases but no correlation seen within controls (IBD $r-0.32, p$ $3.64 \times 10^{-7}$ vs. non-IBD $r-0.14, p=0.77$; TSS1500). There were 6 genes with methylation probes within TSS200 region, all demonstrating negative correlations with DNA methylation apart from SYNE2 (IBD r $0.31, p=2.89 \times 10^{-6}$ vs. non-IBD r $0.22, p=0.02$ ).

\section{Integrative analysis identify immune cell related activation in IBD}

Multi-omics analysis was performed using Multi-omics Factor Analysis (MOFA) ${ }^{25}$. Integration of IBDrelated SNPs and mRNAs around the 137 DMPs produced ten factors, of which the first four explained most of the variability in the dataset (Fig. 3A). Factor1 and Factor 4 strongly correlated with IBD and hsCRP (Fig. 3B\&E). These 2 factors were mostly influenced by DMPs and mRNAs (Fig. 3C) and remained independent of each other ( $r=0.052$, Fig. 3D). Factor1 was higher in IBD compared with controls regardless of inflammation (Fig. 3F; $p<2.20 \times 10^{-16}$ ) and appeared specific for IBD. On the contrary, at null hsCRP, Factor 4 was similar in IBD patients and controls ( $p=0.374$ when $<0.5 \mathrm{mg} / \mathrm{L}$ ) but was found to be elevated in IBD overall (Fig. 3D; $p=4.21 \times 10^{-13}$ ) due to higher values relative to control starting at even marginally increased hsCRP. In UC, Factor1 reflected the extent of colitis (Fig. 3G). Thus, multi-omics integration revealed DMP-driven, IBD- and hsCRP-associated factors 1 and 4.

Factor 1 was defined by greater methylation of genes such as CXCR6 and CD247, and reduced methylation of ZEB2 (Fig. 3H-I). The transcriptomic repertoire of Factor 1 prominently featured S100 proteins and matrix metalloproteinases. Only one IBD GWAS SNP contributed to Factor 1: rs7495132 (CRTC3). In gene ontology, Factor1 was related to inflammation with activation of undifferentiated leukocytes, and lipid metabolism (Fig. 3J).

Reduced methylation at VMP1 (TMEM49) and the interferon-inducible inflammasome trigger AIM2 were driving Factor 4, which also shared lowered ZEB2 methylation with Factor1 (Fig. 3H-I). Gene expression behind Factor 4 included the immunosuppressive CD274 (PD-L1), along with Fc gamma receptor 1 (FCGR1A, FCGR1B) and AIM2 (overexpressed, consistent with lower methylation; Fig. 3J). The GWAS polymorphism rs1801274 (FCGR2A) was negatively correlated with Factor 4. Pathway analysis uncovered potential roles for Factor 4 in immune regulation, lipid metabolism, and cell death (Fig. 3K). The composition of Factor 4 shows that IBD-related differential methylation at VMP1 may relate to opsonization and phagocytosis and testifies of close coupling of pro- and anti-inflammatory responses.

\section{DNA methylation associates with disease course in Inflammatory Bowel Disease}


Follow up data were available for 291 patients with IBD in order to identify methylation markers that predicted treatment escalation in IBD over a median follow-up period of 526 days (IQR:223-775)(Table 4). Thirty nine patients with a diagnosis of CD, 26 patients with $U C$ and 2 with IBD-U required escalation after a median follow-up time of 98 days (IQR:40-229). The median age in this group was 28 years (range: $18-67)$ and $58 \%$ were male $(n=39)$. For downstream analysis, data were split into training $\left(2 / 3^{\text {rd }}\right)$ and testing set $\left(1 / 3^{\text {rd }}\right)$ for signal validation. In order to investigate DMPs that associate with treatment escalation, principal component (PC) analysis using all methylation probes was performed to identify PCs that associate with treatment escalation. In the training set ( $n=194 ; 40$ escalations), 11 PCs significantly associated with treatment escalation (Supplementary Figure 6). The first 2 PCs correlate with cell proportions, however certain PCs such as PC17 only associate with treatment escalation. Probes that represent top/bottom $5 \%$ of variance within this principal component are shown in Supplementary Figure 7.

Probes that represented the top 5\% of variance across all 11PCs that associate with treatment escalation in the training set were selected $(n=55)$ for further LASSO penalised Cox regression. A total of 3 methylation probes remained significant predictors (Table 5) in IBD. These included Transporter associated with Antigen Processing 1 (TAP1; HR: 12.79), Regulatory protein of MTOR complex 1(RPTOR; HR:1.47) and Thymocyte expressed, positive selection associated 1 (TESPA7; HR: 1.29). A model incorporating these 3 probes, was then tested in an independent testing set ( $n=97 ; 27$ escalations). The 3 probe combined model remained a significant predictor of treatment escalation in the testing set with a HR of 5.19 (Cl:2.14-12.56, logrank $p=9.70 \times 10^{-4}$; Figure 4). Adjusting for treatment naivety did not influence the top differentially methylated probes among patients with IBD. Similar analyses were performed to generate a model that included known clinical predictors: age, sex, hsCRP and albumin in the training set $(n=174)$ and validated in the testing set $(n=88)$ where data for covariates were complete. This clinical model had an HR of $4.55\left(\mathrm{Cl}: 2.00-10.35 ; \mathrm{p}=1.03 \times 10^{-4}\right)$ and performed at par with the methylation model. Supplementary Table 16 shows correlations of the top 3 prognostic DMPs with conventional inflammatory markers.

\section{Discussion}

\section{Are geographical replicability and variability preferentially driven by germline variation or alterations in the exposome?}

In complex diseases such as IBD, DNA methylation potentially represents a mechanism at the interface between genetics and the environment. The strengths of this prospective case-control study include recruitment of individuals with a new diagnosis of IBD, mostly naïve to medical therapy, across multiple clinical centres in Europe. Importantly our data strongly replicate, validate and extend previous key DMP findings from children with $C D$ and adults in the index Scottish population where our initial data were generated $^{8,9}$ to Scandinavia. We demonstrate significant evidence for dysregulation of several previously implicated loci, notably VMP1, RPS6KA2 and SBNO2 across Scandinavia and UK, populations with a 
shared genetic ancestry. Replication was less evident in the smaller Southern European cohort recruited in Spain. Despite being adequately powered to detect significant differences in 11 of the 34 probes tested, significant differential methylation for top probes such as ZEB2 and SBNO2 were not seen in the Spanish cohort. RPS6KA2 remains the only consistent signal across UK, Scandinavia and Spain. These differences may be in line with the North-South gradient in IBD that have been reported across USA and Europe $^{26-28}$. Whilst more detailed studies in Southern Europe are needed, these data may suggest population specificity of several methylation changes in IBD and heighten the focus on exploring genetic influences and factors within the exposome in these populations.

DMPs implicated in our study such as VMP1, RPS6KA2 and SBNO2 were also highly significant in the paediatric studies at diagnosis (RISK consortium; USA and Canada) ${ }^{10}$. It remains to be seen whether the methylation differences seen within our predominantly Caucasian non-Jewish cohort are replicated in other non-Caucasian populations. Population-specific methylation differences at $439 \mathrm{CpG}$ sites were reported in a study that included Caucasian-American, African-American and Han Chinese-American healthy individuals and associated these changes with distinct phenotypic characteristics such as drug metabolism, disease susceptibility and appearance ${ }^{29}$. Although, the majority of these differences were due to underlying genetic variations, up to $1 / 3^{\text {rd }}$ of the DMPs were independent of germline variations, bringing into focus the role of non-genetic influences such as the exposome on the epivariance across populations. Similar findings were also reported in a Hispanic origin cohort where genetic ancestry explained up to $3 / 4^{\text {th }}$ of the variance in methylation ${ }^{30}$ and an Indonesian cohort where up to $10 \%$ of genes showed differential expression and methylation patterns between islands, likely attributed to smallscale environmental differences ${ }^{31}$. It remains to be determined whether the differences which are apparent between Northern Europe and Southern Europe within our study are genetically determined or whether these are related to the exposome. Further multiregional epigenome studies are now needed to explore this further in IBD.

\section{The influence of genetics on DNA methylation in IBD}

The differences between populations focus interest on the relative importance of germline variation on methylation; and in turn on gene expression. Our data also shed new light on the relationship between germline variation at specific loci and epigenetic alterations in complex disease. A key locus, VMP1 showed 143 cis-meQTLs across 6 probes and includes meQTLs with a known GWAS snp rs 1292053 and its LD snp( rs8078424, top $\left.p=1.48 \times 10^{-20}\right)^{9}$. However, causal inference using mendelian randomisation could not be determined. A recent study in CD demonstrated that DNA methylation at $3 \mathrm{CpG}$ sites causally associated with a GWAS snp rs1819333 using mendelian randomisation analysis, likely through transcriptional regulation of RPS6KA2 expression ${ }^{10,32}$. This snp was not on our genotyping platform and further validation could not be performed in this study. RPS6KA2 specific hypomethylation has

consistently been demonstrated in IBD across several independent cohorts and across all ages ${ }^{8-10}$. This gene encodes for a ribosomal kinase, a member of the serine/threonine kinase family and regulates the 
autophagy associated mTOR pathway and particularly relevant in $C^{33}$. These data provide insight into the complex interaction of genetics and epigenetics in the pathophysiology of IBD.

\section{Pro-inflammatory pathways}

Of particular interest within the field of DNA methylation has been to address whether the changes seen are causal or a consequence of disease initiation. Somineni et al explored this in the paediatric cohort through several methods including longitudinal dynamics of methylation, correlation with known inflammatory markers and mendelian randomisation ${ }^{10}$. Although the vast majority of methylation signals in the Somineni et al study represented a consequence of disease initiation through inflammation, there were $10 \mathrm{CpGs}$ that were unchanged at follow up ${ }^{34}$. In our studies, some of the methylation alterations have been shown to associate with CRP levels ${ }^{21}$. Even though these changes may be a consequence of inflammation, the genes implicated are of great interest as potential targets for future drug discovery and the mechanisms of how these genes associate with inflammation, disease onset and disease course need further exploration. An example includes OSM, a gene that has been recently shown to be upregulated in inflamed intestinal tissue in IBD and is pro-inflammatory. This gene is able to predict antiTNF response in IBD, making it a useful clinical biomarker and a potential drug target ${ }^{35}$. In our study, a $450 \mathrm{~K}$ probe close to the TSS (TSS1500, $\mathrm{p}=8.6^{\prime} 10^{-11}$ ) of OSM is differentially methylated in IBD compared to controls and demonstrates correlation with OSM gene expression in IBD compared to controls (IBD r -0.32, p $3.64 \times 10^{-7}$ vs. non-IBD $r-0.14, p=0.77$; TSS1500). Other signals linked with CRP-associated DMPs have relevance in IBD pathogenesis. One of the top signals is P-selectin glycoprotein ligand-1(PSGL-1 or $S E L P L G)$ and plays a critical role in immune cell recruitment to sites of tissue inflammation ${ }^{36}$. Anti-PSGL1 antibody is currently in phase 2 drug trial for the treatment of CD (NIH project no. 1R44DK08584501A1). Furthermore, differential methylation within this gene correlated with PSGL-1 expression in our study(IBD $r-0.53, p=2.24 \times 10^{-22}$ vs $-0.37, p=6.34 \times 10^{-8} ; 5^{\prime} U T R$ ). It is known that a normal level of DNA methylation is required to control differential expression of maternal and paternal alleles of imprinted genes ${ }^{37}$ and plays an important role in cell differentiation and embryonic development. Studies have shown that methylation at gene promoter regions can vary depending on cell type with hypermethylation corresponding with low or no gene transcription ${ }^{38,39}$.

Multi-omic data integration using MOFA revealed 2 factors (Factor 1 and 4) strongly associated with IBD and pro-inflammatory pathways. While Factor 1 was defined by greater methylation within CXCR6 and CD247 genes, reduced methylation of ZEB2 (Fig. 3H-I) and mRNA expression predominantly featuring S100 proteins and matrix metalloproteinases, Factor 4 however was defined by reduced methylation at VMP1 (TMEM49) and the interferon-inducible inflammasome trigger AIM2. The CpGs and mRNA repertoire that define these 2 factors highlight the role immune cell activation, cellular response and regulation play in shaping the circulatory methylome and transcriptome in patients with IBD at disease inception. These data provide a repertoire of novel targets to develop future drug therapies that target these pro-inflammatory pathways at disease onset. 


\section{Age acceleration in Inflammatory Bowel Disease}

There is emerging interest in studying disease-associated age acceleration, defined as the difference between predicted age (determined by DNA methylation patterns) and chronological age. Recent studies have linked epigenetic age acceleration to all cause mortality 40,41 and cardiovascular disease outcomes $^{42}$. Epigenetic age acceleration is yet to be explored in the context of immune-mediated diseases such as IBD. In our cohort, significant DiffAge was seen between IBD and controls (non-IBD: median 4.34 years (IQR:3.83-4.70) vs. IBD: 5.28 years(IQR: 4.72-5.64); $\mathrm{p}<2.20 \times 10^{-16}$ ); this was consistently seen within CD and UC when compared to non-IBD (vs. UC: 5.08 years(IQR: 4.51-5.49); $\mathrm{p}<2.20 \times 10^{-16}$ and vs. $C D$ : 5.53 years(IQR: 4.99-5.79); $\left.\mathrm{p}<2.20 \times 10^{-16}\right)$. Of particular interest was the absence of its association with drug exposure both in CD and UC (UC $p=0.89, C D p=0.24)$ and poor correlation with inflammatory indices at disease inception. Further studies measuring serial methylation profiles may help determine whether these differences persist after treatment, over time.

\section{The epigenome of treatment escalation}

Of clinical interest, DNA methylation alterations were investigated for their performance as prognostic markers. A unique strength of our study lies in the evaluation of methylation in predicting an aggressive disease course in a treatment-naïve unselected inception cohort. We identify a simple molecular signature worthy of further attention as a potential predictive biomarker. The reported prognostic signals have relevance in the pathogenesis of IBD. TAP1 is essential in HLA class I proteins for generation of cytotoxic T-cell mediated immune response. Differential expression of this gene has been shown to associate with immune escape and poor prognosis in colorectal cancer and an indicator of an aggressive breast tumour $^{43,44}$ (Table 5). In IBD, TAP1 differential methylation has been previously reported as a CD specific signal in IECs from small intestine and colon and reported to be a regulatory DMR (rDMR: differentially methylated region (DMRs) that is located within $10 \mathrm{~kb}$ of the transcription start site of a differentially expressed gene) ${ }^{20}$. RPTOR gene forms part of the mTORC1 complex which has been shown to promote UC through COX-2 mediated Th17 responses ${ }^{45}$. Depletion of RPTOR inactivates mTORC1 and ameliorates $U C^{45}$. These differentially methylated genes also differ from those implicated in disease susceptibility. Similar trends have been seen in GWAS in CD where unique prognostic genes have been identified that do not overlap with disease susceptibility ${ }^{14}$.

Several studies including GWAS have utilised the $\mathrm{CD} 8^{+} \mathrm{T}$-cell derived transcriptome criteria for escalation, defined as the need for 2 or more immunosuppressants' after initial disease remission ${ }^{13,14,46-48}$. These studies collectively have provided the first lines of evidence for a circulating biomarker profile to define a subset of patients that require treatment escalation who perhaps may benefit from a 'top-down' approach to management at an early stage. Our data complement these findings and provide further molecular depth by defining their methylome signature. 
Within the paediatric CD group, DNA methylation in separated circulating $C D 8^{+} T$ cells has been shown not to predict outcomes in $\mathrm{CD}^{49}$. Given that $\mathrm{CD} 8^{+}$cells only represent a small proportion of the cellular compartment in whole blood, our signals warrant further exploration of the prognostic methylome in other immune cells. It is noteworthy in this context, the e-FORGE analysis in our dataset preferentially implicates monocytes. Furthermore, our prognostic analysis includes UC; the molecular prognostic profile of this subtype has not been examined previously. Further large multicentre studies are now needed to explore these findings further both in adults and children.

\section{Strengths and limitations}

Our study has recruited over four years one of the largest multi-centre European inception cohorts reported to date, and generated IBD specific methylation signals, associated with disease onset and progression. We have explored geographical, genetic and non-genetic correlates as mechanistic as well as translational implications. We have previously demonstrated that the most dysregulated areas implicated are cell-specific ${ }^{9}$. There are certain methodological considerations to take into account when interpreting our data. Firstly, our dataset is adequately powered in Scandinavia and UK, but we acknowledge that the samples size will detect some, but not all DMPs in Southern Europe (Spain). We have demonstrated, in the Spanish cohort, replication for RPS6KA2, one of the most robust findings in Northern Europe and North America, whilst excluding a significant effect for ten other markers all highly significant in Scandinavia.

One of the challenges of methylation studies in inflammatory diseases is to disentangle the effect of inflammation on the top differentially expressed DMPs. We have approached this in appropriate detail by several methods including correlation analyses with inflammatory markers such as hsCRP and matching our top probes with publicly available inflammation-associated DMPs.

There are also potential challenges in the prognostic analysis of data from multi-centre studies associated with the concern that decision-making can vary across centres. However, in our study all European centres used similar criteria and guidelines for decisions on escalation in IBD (also known as 'step-up' approach) where increments are made in therapy based on response to the initial treatment. Although we were able to identify and internally validate IBD-specific prognostic signals in this analysis, IBD subtype analysis and validation was not feasible due to the small number of patients who escalated therapy within each subtype. It is worth noting, however, that certain prognostic signals such as TAP1 have been shown to be CD specific in the published literature. Future studies are needed to further validate our findings.

\section{Conclusions}

This study highlights the stability of the IBD-specific circulating methylome across regions with shared ancestry. We demonstrate a close association of the methylome with inflammation and through integrative multi-omic analyses we identify key pro-inflammatory genes that are upregulated in IBD at 
inception. Furthermore, differential methylation within certain genes such as TAP1 associate with disease course over time. These data provide a rich resource for future translational studies investigating the epigenome in IBD; and potentially represent a paradigm for analysis in other complex diseases.

\section{Methods}

\section{Study design}

Patients were recruited prospectively as part of the IBD-CHARACTER inception cohort (reference 305676) from gastroenterology appointments across 7 centres in Europe (Table 1). All IBD cases met the standard diagnostic criteria for either UC or CD following thorough clinical, microbiological, endoscopic, histological, and radiological evaluation. The Lennard-Jones, Montreal and Paris criteria were used for diagnosis and classification of clinical phenotypes. The control group consisted of symptomatic controls attending gastroenterology clinic during the same period with no evidence of IBD after further investigations and at follow up. Healthy volunteers were also recruited into the study.

We collected patient demographics including sex, age at diagnosis, and date of diagnosis. Details of drug therapy and concomitant medications were recorded. Treatment naïvety within the IBD cohort was defined as no exposure to any IBD-related medical therapies such as oral and topical steroids, oral and topical 5-ASA therapies, biological therapies and immunomodulators. High-sensitivity CRP (hsCRP), albumin were re-assayed in a single batch at the end of recruitment. Other routine markers including haemoglobin, white cell count were also recorded.

Patients with IBD were followed prospectively and information on clinical outcomes were collected during follow-up. Treatment escalation was defined as the need for a biologic, ciclosporin or surgery, instituted for disease flare after initial induction therapy and aiming to induce disease remission. In UC, the definition of treatment escalation also included colectomy during index admission.

All patients and controls provided written, informed consent with local ethical approval at each centre.

\section{Genome-wide methylation profiling}

Peripheral blood leukocyte DNA was bisulphite converted and analysed using the Illumina HumanMethylation450 platform (IIlumina, San Diego, CA, USA). ${ }^{50}$ Cases and controls were randomly distributed across chips. Data were processed using the meffil package ${ }^{51}$ in $\mathrm{R}$ (R Foundation for Statistical Computing, Vienna). Samples containing $>1 \%$ probes with detection $p$ values $>0.01$ were removed. Probes with bead counts $<3$ in $10 \%$ samples, or detection p values $>0.01$ in $10 \%$ samples were also removed. Sex mismatches were identified by analysing the median intensities of the sex chromosome probes and removed from further analyses. Genotypes were compared with genotyping probes on the methylation array. Probes containing snps with a minor allele frequency of $\geq 0.01$ in the European population in the 1000 Genomes Project were also removed. 
Three samples with low signal intensities were removed ( $>1 \%$ probes with detection $p$ values $>0.01)$ and 684 probes were filtered out due to low signal. There were $n=15$ failed quality control that were removed from further analyses.

\section{Differentially methylated positions and regions}

Cell proportions were estimated from methylation data using the Houseman algorithm ${ }^{52}$ using the meffil package in R. Differentially methylated position analysis (DMP, single $\mathrm{CpG}$ probe) was performed using age, sex and cell proportions as covariates ${ }^{53}$. Statistical significance was set at $p<0.05$ following adjustment for multiple testing using Holm correction ${ }^{54}$ for whole blood data.

Differentially methylated regions (DMRs) were identified using the Lasso function from the ChaMP pipeline ${ }^{55,56}$ (distinct from the lasso function described below in the biomarker discovery section) and defined as three or more contiguous probes within a distance threshold based on expected probe density, each achieving a FDR corrected $\mathrm{p}<0.05$ in DMP analysis.

\section{Epigenetic clock and Age acceleration}

Age acceleration (AgeAccel) is defined as the residual resulting from a linear regression model, regressing the Horvath estimate of epigenetic age (biological age) on chronological age ${ }^{7}$. A positive value for AgeAccel indicates that the observed epigenetic age is higher than that predicted, based on chronological age. DiffAge according to Horvath was defined as the differences between predicted biological age and chronological age ${ }^{7}$. A positive value for DiffAge is seen for individuals with an increased biological age compared to their chronological age ${ }^{23}$.

\section{Methylation Quantitative Trait Loci Analyses (MeQTL) and Mendelian Randomisation}

Whole blood leukocyte DNA were extracted using the Nucleon BACC 3 DNA extraction kit (GE Healthcare, Buckinghamshire, UK). Patients were genotyped using the Illumina HumanOmniExpressExome-8 Bead Chips (Illumina, San Diego, CA, USA). A sex-check was performed using PLINK to identify and remove sexmismatches. MeQTLs and eQTLs were estimated using the Matrix-eQTL package ${ }^{57}$, with a distance threshold of $1 \mathrm{Mb}$. snps with a minor allele frequency of $<5 \%$ were filtered from downstream analyses. Age and sex were included as covariates in order to identify specific disease-associated meQTLs. A Holm corrected $p<0.05$ was used as the threshold for statistical significance for disease associated meQTLs.

Mendelian randomisation was performed using TwoSampleMR to determine causal inference ${ }^{24}$ for the top meQTLs. For this analysis meQTLs, independent of IBD were obtained, using the same filtering thresholds as described above. MeQTL snps were used as the instrumental variable, $\mathrm{CpG}$ as the exposure, and IBD as the outcome variable.

\section{Gene expression profiling}


Whole blood RNA underwent targeted RNA sequencing was performed using Ion AmpliSeq Human Gene Expression Core Panel, containing 20,802 genes. Quality control was performed using the lon Library Taqman $^{\mathrm{TM}}$ Quantitation kit. Sequence reads were aligned using the Torrent Suite Software (TSS) and the number of matches per amplicon were quantified. After filtering, 14,182 transcripts were available for further analysis.

\section{Cell-of-origin analysis}

We used eFORGE v2.0 (http://eforge.cs.ucl.ac.uk/) to identify if the replicated CpGs were enriched in DNase I hypersensitive sites (DHSs) (markers of active regulatory regions) and loci with overlapping histone modifications (H3Kme1, H3Kme4, H3K9me3, H3K27me3, and H3K36me3) across available cell lines and tissues from Roadmap Epigenomics Project, BLUEPRINT Epigenome, and ENCODE (Encyclopedia of DNA Elements) consortia data.

\section{Data integrative and pathway analysis}

Integration of genomic, methylomic, and transcriptomic layers was done with Multi-Omics Factor Analysis v2 (MOFA). The following data were selected for cross-omics integration: all the 137 probes differentially methylated between IBD and controls, all the GWAS-related single nucleotide polymorphisms with minor allele frequency > 5\% (SNPs, $n=99)$, and all the available IBD-related transcripts (based on DisGeNET, $n=1021$ ). The integration was conducted using Multi-Omics Factor Analysis v2 (MOFA+) ${ }^{25}$. Prioritized features were subject to gene ontology analysis using gene set enrichment analysis from the Broad Institute (Cambridge, USA). Further details are available in supplementary methods.

\section{Prognostication analysis}

For IBD prognostic marker analysis, data were randomly split to training $\left(2 / 3^{\text {rd }}\right)$ and testing set $\left(1 / 3^{\text {rd }}\right)$. Unsupervised principal component analyses were performed to examine correlates of treatment escalation and clinical predictors of disease severity using the PCAtools package(https://github.com/kevinblighe/PCAtools). Principal components (PC) that associate with treatment escalation were selected to identify methylation probes that explain the top $5 \%$ variance within each PC. These methylation probes were then selected to fit a model using least absolute shrinkage and selection operator (LASSO) penalised Cox regression with age and sex as covariates and 1000 cross validated iterations. Results of the Cox regression were then validated in the testing cohort by generating a combined model using all significant variables. Hazard ratios (HRs) were calculated from Cox regression coefficients. We then performed survival analyses based on the need for treatment escalation as defined above. Comparison for treatment escalation was performed using a log-rank test (1df) and graphically represented with a Kaplan-Meier plot. The performance of the methylation model was also assessed in the IBD subtypes. Conventional predictors of treatment escalation including age, sex, hsCRP and albumin in CD and age, sex, hsCRP, albumin and extent of colitis in UC were also examined. 


\section{Declarations}

Author Contributions: Study design RK, JH, MDA, MV, JS. Patient recruitment and sample processing NTV, RK, NAK, DB, SV, ATA CO, FG, JSO, MP, PR. Experimental work OA, FH, CP, RK, NTV, ATA, NAK, JKN. Data Analysis RK, ATA, JKN. RK, ATA and JKN wrote the manuscript. All authors were involved in critical review, editing, revision and approval of the final manuscript.

Conflict of interest: R. Kalla Financial support for research: EC IBD-Character, Lecture fee(s): Ferring, N. Kennedy Financial support for research: Wellcome Trust, Conflict with: Abbvie, MSD, Warner Chilcott, Ferring speaker fees. Shire Travel bursary, A. Adams: None Declared, J. Satsangi Financial support for research: EC grant IBD-BIOM,IBD-Character, Wellcome, CSO, MRC, Conflict with: Consultant for: Takeda, Conflict with: MSD speaker fees. Shire travelling expenses

Funding: The study has been funded by the following EU FP7 grant: IBD-CHARACTER (contract \# 2858546). NAK was funded by the Wellcome Trust (grant number WT097943MA).

Corresponding authors: Professor J Satsangi, Dr Rahul Kalla, Dr Alex Adams.

\section{References}

1. Kaplan GG, Windsor JW. The four epidemiological stages in the global evolution of inflammatory bowel disease. Nat Rev Gastroenterol Hepatol 2021; 18: 56-66.

2. Hansen R, Russell RK, Reiff $C$ et al. Microbiota of De-Novo Pediatric IBD: Increased Faecalibacterium Prausnitzii and Reduced Bacterial Diversity in Crohn's But Not in Ulcerative Colitis. Am J Gastroenterol 2012;: 1-10.

3. Cedar H, Bergman Y. Linking DNA methylation and histone modification: patterns and paradigms. Nat Rev Genet 2009; 10: 295-304.

4. Kraiczy J, Nayak K, Ross A et al. Assessing DNA methylation in the developing human intestinal epithelium: potential link to inflammatory bowel disease. Mucosal Immunol 2016; 9: 647-58.

5. Jones PA. Functions of DNA methylation: islands, start sites, gene bodies and beyond. Nat Rev Genet 2012; 13: 484-492.

6. Tsaprouni LG, Yang T-P, Bell J et al. Cigarette smoking reduces DNA methylation levels at multiple genomic loci but the effect is partially reversible upon cessation. Epigenetics 2014; 9: 1382-96.

7. Horvath S. DNA methylation age of human tissues and cell types. Genome Bio/ 2013; 14: R115.

8. Adams AT, Kennedy NA, Hansen R et al. Two-stage genome-wide methylation profiling in childhoodonset Crohn's Disease implicates epigenetic alterations at the VMP1/MIR21 and HLA loci. Inflamm Bowel Dis 2014; 20: 1784-93.

9. Ventham NT, Kennedy NA, Adams AT et al. Integrative epigenome-wide analysis demonstrates that DNA methylation may mediate genetic risk in inflammatory bowel disease. Nat Commun 2016; 7: 13507. 
10. Somineni HK, Venkateswaran S, Kilaru V et al. Blood-derived DNA Methylation Signatures of Crohn's Disease and Severity of Intestinal Inflammation. Gastroenterology 2019. doi:10.1053/j.gastro.2019.01.270.

11. Kugathasan S, Denson LA, Walters TD et al. Prediction of complicated disease course for children newly diagnosed with Crohn's disease: a multicentre inception cohort study. Lancet (London, England) 2017; 389: 1710-1718.

12. Marigorta UM, Denson LA, Hyams JS et al. Transcriptional risk scores link GWAS to eQTLs and predict complications in Crohn's disease. Nat Genet 2017; 49: 1517-1521.

13. Kalla R, Kennedy NA, Ventham NT et al. Serum Calprotectin: A Novel Diagnostic and Prognostic Marker in Inflammatory Bowel Diseases. Am J Gastroenterol 2016; 111: 1796-1805.

14. Lee JC, Biasci D, Roberts $R$ et al. Genome-wide association study identifies distinct genetic contributions to prognosis and susceptibility in Crohn's disease. Nat Genet 2017; 49: 262-268.

15. Miyahara K, Nouso K, Saito $S$ et al. Serum glycan markers for evaluation of disease activity and prediction of clinical course in patients with ulcerative colitis. PLoS One 2013; 8: e74861.

16. Biasci D, Lee JC, Noor NM et al. A blood-based prognostic biomarker in IBD. Gut 2019; 68: 13861395.

17. Verstockt B, Verstockt S, Dehairs J et al. Low TREM1 expression in whole blood predicts anti-TNF response in inflammatory bowel disease. EBioMedicine 2019; 40: 733-742.

18. Gaujoux R, Starosvetsky E, Maimon N et al. Cell-centred meta-analysis reveals baseline predictors of anti-TNFa non-response in biopsy and blood of patients with IBD. Gut 2019; 68: 604-614.

19. Breeze CE, Reynolds AP, van Dongen J et al. eFORGE v2.0: updated analysis of cell type-specific signal in epigenomic data. Bioinformatics 2019; 35: 4767-4769.

20. Howell KJ, Kraiczy J, Nayak KM et al. DNA Methylation and Transcription Patterns in Intestinal Epithelial Cells From Pediatric Patients With Inflammatory Bowel Diseases Differentiate Disease Subtypes and Associate With Outcome. Gastroenterology 2017. doi:10.1053/j.gastro.2017.10.007.

21. Ligthart S, Marzi C, Aslibekyan S et al. DNA methylation signatures of chronic low-grade inflammation are associated with complex diseases. Genome Biol 2016; 17: 255.

22. Gao X, Jia M, Zhang Y, Breitling LP, Brenner H. DNA methylation changes of whole blood cells in response to active smoking exposure in adults: a systematic review of DNA methylation studies. Clin Epigenetics 2015; 7: 113.

23. Lind L, Ingelsson E, Sundström J, Siegbahn A, Lampa E. Methylation-based estimated biological age and cardiovascular disease. Eur J Clin Invest 2018; 48: e12872.

24. Hemani G, Zheng J, Elsworth B et al. The MR-Base platform supports systematic causal inference across the human phenome. Elife 2018; 7. doi:10.7554/eLife.34408.

25. Argelaguet R, Arnol D, Bredikhin D et al. MOFA+: a statistical framework for comprehensive integration of multi-modal single-cell data. Genome Biol 2020; 21: 111. 
26. Shivananda S, Lennard-Jones J, Logan R et al. Incidence of inflammatory bowel disease across Europe: is there a difference between north and south? Results of the European Collaborative Study on Inflammatory Bowel Disease (EC-IBD). Gut 1996; 39: 690-7.

27. Sonnenberg A, McCarty DJ, Jacobsen SJ. Geographic variation of inflammatory bowel disease within the United States. Gastroenterology 1991; 100: 143-9.

28. Armitage EL, Aldhous MC, Anderson N et al. Incidence of juvenile-onset Crohn's disease in Scotland: association with northern latitude and affluence. Gastroenterology 2004; 127: 1051-7.

29. Heyn H, Moran S, Hernando-Herraez I et al. DNA methylation contributes to natural human variation. Genome Res 2013; 23: 1363-1372.

30. Galanter JM, Gignoux CR, Oh SS et al. Differential methylation between ethnic sub-groups reflects the effect of genetic ancestry and environmental exposures. Elife 2017; 6. doi:10.7554/eLife.20532.

31. Natri HM, Bobowik KS, Kusuma P et al. Genome-wide DNA methylation and gene expression patterns reflect genetic ancestry and environmental differences across the Indonesian archipelago. PLOS Genet 2020; 16: e1008749.

32. Wu Y, Zeng J, Zhang $F$ et al. Integrative analysis of omics summary data reveals putative mechanisms underlying complex traits. Nat Commun 2018; 9: 918.

33. Pancholi S, Lykkesfeldt AE, Hilmi C et al. ERBB2 influences the subcellular localization of the estrogen receptor in tamoxifen-resistant MCF-7 cells leading to the activation of AKT and RPS6KA2. Endocr Relat Cancer 2008; 15: 985-1002.

34. Kalla R, Adams AT, Satsangi J. Blood-based DNA methylation in Crohn's disease and severity of intestinal inflammation. Trans/ Gastroenterol Hepatol 2019; 4: 76-76.

35. West NR, Hegazy AN, Owens BMJ et al. Oncostatin M drives intestinal inflammation and predicts response to tumor necrosis factor-neutralizing therapy in patients with inflammatory bowel disease. Nat Med 2017; 23: 579-589.

36. Guyer DA, Moore KL, Lynam EB et al. P-selectin glycoprotein ligand-1 (PSGL-1) is a ligand for Lselectin in neutrophil aggregation. Blood 1996; 88: 2415-21.

37. Li E, Beard C, Jaenisch R. Role for DNA methylation in genomic imprinting. Nature 1993; 366: 362-5.

38. Suzuki MM, Bird A. DNA methylation landscapes: provocative insights from epigenomics. Nat Rev Genet 2008; 9: 465-76.

39. Han H, Cortez CC, Yang X, Nichols PW, Jones PA, Liang G. DNA methylation directly silences genes with non- $\mathrm{CpG}$ island promoters and establishes a nucleosome occupied promoter. Hum Mol Genet 2011; 20: 4299-310.

40. Marioni RE, Shah S, McRae AF et al. DNA methylation age of blood predicts all-cause mortality in later life. Genome Biol 2015; 16: 25.

41. Chen BH, Marioni RE, Colicino E et al. DNA methylation-based measures of biological age: metaanalysis predicting time to death. Aging (Albany NY) 2016; 8: 1844-1865. 
42. Roetker NS, Pankow JS, Bressler J, Morrison AC, Boerwinkle E. Prospective Study of Epigenetic Age Acceleration and Incidence of Cardiovascular Disease Outcomes in the ARIC Study (Atherosclerosis Risk in Communities). Circ Genomic Precis Med 2018; 11: e001937.

43. Ling A, Löfgren-Burström A, Larsson $P$ et al. TAP1 down-regulation elicits immune escape and poor prognosis in colorectal cancer. Oncoimmunology 2017; 6: e1356143.

44. Henle AM, Nassar A, Puglisi-Knutson D, Youssef B, Knutson KL. Downregulation of TAP1 and TAP2 in early stage breast cancer. PLoS One 2017; 12: e0187323.

45. Lin X, Sun Q, Zhou L et al. Colonic epithelial mTORC1 promotes ulcerative colitis through COX-2mediated Th17 responses. Mucosal Immunol 2018; 11: 1663-1673.

46. Lee JC, Lyons $\mathrm{P}$ a, McKinney EF et al. Gene expression profiling of CD8 + T cells predicts prognosis in patients with Crohn disease and ulcerative colitis. J Clin Invest 2011; 121: 4170-9.

47. Kalla R, Adams AT, Bergemalm D et al. Serum proteomic profiling at diagnosis predicts clinical course, and need for intensification of treatment in inflammatory bowel disease. J Crohns Colitis 2020. doi:10.1093/ecco-jcc/jjaa230.

48. Kalla R, Adams AT, Ventham NT et al. Whole blood profiling of T-cell derived miRNA allows the development of prognostic models in inflammatory bowel disease. J Crohns Colitis 2020. doi:10.1093/ecco-jcc/jjaa134.

49. Gasparetto M, Payne F, Nayak K et al. Transcription and DNA Methylation patterns of blood derived $\mathrm{CD} 8+\mathrm{T}$ cells are associated with age and Inflammatory Bowel Disease but do not predict prognosis. Gastroenterology 2020. doi:10.1053/j.gastro.2020.08.017.

50. Bibikova M, Barnes B, Tsan $C$ et al. High density DNA methylation array with single CpG site resolution. Genomics 2011; 98: 288-95.

51. Min JL, Hemani G, Davey Smith G, Relton C, Suderman M. Meffil: efficient normalization and analysis of very large DNA methylation datasets. Bioinformatics 2018. doi:10.1093/bioinformatics/bty 476 .

52. Houseman EA, Accomando WP, Koestler DC et al. DNA methylation arrays as surrogate measures of cell mixture distribution. BMC Bioinformatics 2012; 13: 86.

53. Smyth GK. Limma: linear models for microarray data. In: Gentleman R, Carey V, Dudoit S, Irizarry R, Huber W (eds). Bioinformatics and Computational Biology Solutions Using $\{R\}$ and Bioconductor. Springer: New York, 2005, pp 397-420.

54. Holm S. A simple sequentially rejective multiple test procedure. Scand J Stat 1979; 6: 65-70.

55. Morris TJ, Butcher LM, Feber A et al. ChAMP: 450k Chip Analysis Methylation Pipeline. Bioinformatics 2014; 30: 428-30.

56. Aryee MJ, Jaffe AE, Corrada-Bravo $\mathrm{H}$ et al. Minfi: a flexible and comprehensive Bioconductor package for the analysis of Infinium DNA methylation microarrays. Bioinformatics 2014; 30: 1363-9.

57. Shabalin AA. Matrix eQTL: ultra fast eQTL analysis via large matrix operations. Bioinformatics 2012; 28: $1353-8$. 
58. Wang $D$, Zheng $M$, Lei $L$ et al. Tespa1 is involved in late thymocyte development through the regulation of TCR-mediated signaling. Nat Immunol 2012; 13: 560-8.

59. Ha KCH, Sterne-Weiler T, Morris Q, Weatheritt RJ, Blencowe BJ. Differential contribution of transcriptomic regulatory layers in the definition of neuronal identity. Nat Commun 2021; 12: 335.

60. Waszak SM, Robinson GW, Gudenas BL et al. Germline Elongator mutations in Sonic Hedgehog medulloblastoma. Nature 2020; 580: 396-401.

61. de Lange KM, Moutsianas L, Lee JC et al. Genome-wide association study implicates immune activation of multiple integrin genes in inflammatory bowel disease. Nat Genet 2017; 49: 256-261.

\section{Tables}

Table 1: Study demographics and study recruitment according to clinical centre 


\begin{tabular}{|c|c|c|}
\hline \multicolumn{3}{|l|}{ Study demographics } \\
\hline Variables & $\begin{array}{l}\text { Inflammatory Bowel Diseases } \\
(\mathrm{n}=343)\end{array}$ & $\begin{array}{l}\text { Controls } \\
(\mathrm{n}=295)\end{array}$ \\
\hline Males (\%) & $185(54)$ & $130(44)$ \\
\hline Subtype IBD (CD:UC:IBD-U) & 154:161:28 & \\
\hline Subtype Controls (HC: Non-IBD) & & $54: 241$ \\
\hline Smoking status (current: never: ex: missing) & 54:147:103:39 & $53: 147: 58: 37$ \\
\hline Mean age (range) & $34(7-79)$ & $33(3-79)$ \\
\hline \multicolumn{2}{|l|}{ Montreal classification for CD } & \\
\hline L1 & $48,1(31 \%, 1 \%)$ & \\
\hline $\mathrm{L} 2,+\mathrm{L} 4$ & $41,5(27 \%, 3 \%)$ & \\
\hline $\mathrm{L} 3,+\mathrm{L} 4$ & $46,10(30 \%, 6 \%)$ & \\
\hline Isolated L4 & $3(2 \%)$ & \\
\hline \multicolumn{2}{|l|}{ Montreal Behaviour for CD } & \\
\hline $\begin{array}{l}\text { B1, B1p (non-stricturing \& non-penetrating, } \\
\text { +perianal) }\end{array}$ & $120,6(78 \%, 4 \%)$ & \\
\hline B2, B2p (stricturing, +perianal) & $12,0(8 \%, 0 \%)$ & \\
\hline B3, B3p (penetrating, +perianal) & $6,6(4 \%, 4 \%)$ & \\
\hline Not available & $4(2 \%)$ & \\
\hline \multicolumn{2}{|l|}{ Paris Extent for UC } & \\
\hline E1 & $41(25 \%)$ & \\
\hline E2 & $52(32 \%)$ & \\
\hline E3 & $67(42 \%)$ & \\
\hline Not available & $1(1 \%)$ & \\
\hline
\end{tabular}




\begin{tabular}{|llllll|}
\hline Centre & $\mathrm{CD}$ & $\mathrm{UC}$ & IBD-U & Controls & Total \\
Edinburgh & 47 & 57 & 10 & 86 & 200 \\
Linkoping & 11 & 4 & 1 & 41 & 57 \\
Maastricht & 4 & 1 & 0 & 0 & 5 \\
Orebro & 24 & 21 & 9 & 65 & 119 \\
Oslo & 54 & 68 & 6 & 63 & 191 \\
IACS & 14 & 10 & 2 & 40 & 66 \\
Total & 154 & 161 & 28 & 295 & 638 \\
\hline
\end{tabular}

Footnote: Numbers denote the recruitment figures per centre within the study

Table 2: Top 10 differentially methylation positions (DMPs) in patients with Inflammatory Bowel Diseases versus controls. 


\begin{tabular}{|c|c|c|c|c|c|c|c|}
\hline Probe ID & $\mathrm{Chr}$ & Gene ID & $\begin{array}{l}\text { Log } \\
\text { FC }\end{array}$ & $P$ value & Holm P & Region & $\begin{array}{c}\text { Relation to CpG } \\
\text { island }\end{array}$ \\
\hline $\operatorname{cg} 07573872$ & 19 & SBNO2 & -0.05 & $\begin{array}{r}3.07 \mathrm{E}- \\
23\end{array}$ & $\begin{array}{r}1.05 \mathrm{E}- \\
17\end{array}$ & Body & S_Shelf \\
\hline $\operatorname{cg} 09349128$ & 22 & CRELD2 & -0.03 & $\begin{array}{r}3.87 \mathrm{E}- \\
21\end{array}$ & $\begin{array}{r}1.32 \mathrm{E}- \\
15\end{array}$ & & N_Shore \\
\hline $\operatorname{cg} 17501210$ & 6 & RPS6KA2 & -0.04 & $\begin{array}{r}1.25 \mathrm{E}- \\
19\end{array}$ & $\begin{array}{r}4.26 \mathrm{E}- \\
14\end{array}$ & Body & OpenSea \\
\hline $\operatorname{cg} 12054453$ & 17 & VMP1 & -0.07 & $\begin{array}{r}4.89 \mathrm{E}- \\
19\end{array}$ & $\begin{array}{r}1.67 \mathrm{E}- \\
13\end{array}$ & Body & OpenSea \\
\hline $\operatorname{cg} 16292768$ & 8 & CLU & -0.03 & $\begin{array}{r}4.85 \mathrm{E}- \\
18\end{array}$ & $\begin{array}{r}1.66 \mathrm{E}- \\
12\end{array}$ & Body & OpenSea \\
\hline $\operatorname{cg} 18608055$ & 19 & SBNO2 & -0.04 & $\begin{array}{r}8.67 \mathrm{E}- \\
17\end{array}$ & $\begin{array}{r}2.96 \mathrm{E}- \\
11\end{array}$ & Body & OpenSea \\
\hline $\operatorname{cg} 16936953$ & 17 & VMP1 & -0.07 & $\begin{array}{r}2.32 \mathrm{E}- \\
16\end{array}$ & $\begin{array}{r}7.91 \mathrm{E}- \\
11\end{array}$ & Body & OpenSea \\
\hline cg19821297 & 19 & HOOK2 & -0.04 & $\begin{array}{r}5.32 \mathrm{E}- \\
16\end{array}$ & $\begin{array}{r}1.81 \mathrm{E}- \\
10\end{array}$ & & S_Shore \\
\hline $\operatorname{cg} 27469606$ & 19 & SBNO2 & -0.03 & $\begin{array}{r}1.09 \mathrm{E}- \\
15\end{array}$ & $\begin{array}{r}3.73 \mathrm{E}- \\
10\end{array}$ & 5 'UTR & Island \\
\hline $\operatorname{cg} 12170787$ & 19 & SBNO2 & -0.02 & $\begin{array}{r}4.29 \mathrm{E}- \\
15\end{array}$ & $\begin{array}{r}1.47 \mathrm{E}- \\
09\end{array}$ & Body & OpenSea \\
\hline
\end{tabular}

Footnote: Positive value indicated increased methylation in cases compared to controls, negative values indicated hypomethylation in IBD cases versus controls. NA denotes methylation probes with no annotated gene symbol. Body: within body of the gene. 5'UTR: 5'untranslated region 
Table 3: Top 10 differentially methylation positions (DMPs) in patients with Inflammatory Bowel Diseases versus controls that were tested in the Scandinavian cohort (NE Holm represents holm corrected $\mathrm{p}$ value) and replicated across UK (UK Holm represents $\mathrm{p}$ value) and Sain (SE Holm). The highlighted probe remains significant across all cohorts.

\begin{tabular}{|c|c|c|c|c|c|c|c|c|c|}
\hline \multirow[t]{2}{*}{ Probe ID } & \multirow[t]{2}{*}{$\mathrm{Chr}$} & \multirow[t]{2}{*}{ Gene } & \multirow[t]{2}{*}{ Region } & \multirow{2}{*}{$\begin{array}{l}\text { Relation to } \\
\text { CpG Island }\end{array}$} & \multirow{2}{*}{$\begin{array}{l}\text { Log } \\
\text { FC }\end{array}$} & \multirow{2}{*}{$\begin{array}{l}\text { NE P } \\
\text { value }\end{array}$} & \multirow{2}{*}{$\begin{array}{l}\mathrm{NE} \\
\text { Holm }\end{array}$} & \multirow{2}{*}{$\begin{array}{l}\text { UK } \\
\text { Holm }\end{array}$} & \multirow{2}{*}{$\begin{array}{l}\text { SE } \\
\text { Holm }\end{array}$} \\
\hline & & & & & & & & & \\
\hline \multirow[t]{2}{*}{$\operatorname{cg} 17501210$} & 6 & RPS6KA2 & Body & OpenSea & -0.04 & $4.61 \mathrm{E}-$ & $1.57 \mathrm{E}-$ & $5.26 \mathrm{E}-$ & $2.51 \mathrm{E}-$ \\
\hline & & & & & & 09 & 03 & 08 & 02 \\
\hline \multirow[t]{2}{*}{$\operatorname{cg} 12992827$} & 3 & ZLPD1 & & OpenSea & -0.05 & $1.81 \mathrm{E}-$ & $6.18 \mathrm{E}-$ & $9.89 \mathrm{E}-$ & $2.13 \mathrm{E}-$ \\
\hline & & & & & & 09 & 04 & 04 & 01 \\
\hline \multirow[t]{2}{*}{ cg02464912 } & 14 & SYNE2 & TSS200 & N_Shore & -0.01 & $7.46 \mathrm{E}-$ & $2.55 \mathrm{E}-$ & $1.00 \mathrm{E}-$ & $3.28 \mathrm{E}-$ \\
\hline & & & & & & 08 & 02 & 01 & 01 \\
\hline \multirow[t]{2}{*}{$\operatorname{cg} 12054453$} & 17 & VMP1 & Body & OpenSea & -0.07 & $2.02 \mathrm{E}-$ & $6.89 \mathrm{E}-$ & $2.37 \mathrm{E}-$ & $5.08 \mathrm{E}-$ \\
\hline & & & & & & 13 & 08 & 04 & 01 \\
\hline \multirow[t]{2}{*}{$\operatorname{cg} 01101459$} & 1 & LINC01132 & & OpenSea & 0.03 & $3.78 \mathrm{E}-$ & $1.29 \mathrm{E}-$ & $5.72 \mathrm{E}-$ & $5.08 \mathrm{E}-$ \\
\hline & & & & & & 08 & 02 & 02 & 01 \\
\hline \multirow[t]{2}{*}{$\operatorname{cg} 02782634$} & 17 & VMP1 & Body & OpenSea & -0.02 & $1.04 \mathrm{E}-$ & $3.56 \mathrm{E}-$ & $1.00 \mathrm{E}-$ & $5.55 \mathrm{E}-$ \\
\hline & & & & & & 07 & 02 & 01 & 01 \\
\hline \multirow[t]{2}{*}{$\operatorname{cg} 18942579$} & 17 & VMP1 & Body & OpenSea & -0.05 & $1.77 \mathrm{E}-$ & $6.03 \mathrm{E}-$ & $8.90 \mathrm{E}-$ & $5.55 \mathrm{E}-$ \\
\hline & & & & & & 08 & 03 & 05 & 01 \\
\hline \multirow[t]{2}{*}{$\operatorname{cg} 09349128$} & 22 & CRELD2 & & N_Shore & -0.04 & $3.80 \mathrm{E}-$ & $1.30 \mathrm{E}-$ & $3.07 \mathrm{E}-$ & $6.88 \mathrm{E}-$ \\
\hline & & & & & & 17 & 11 & 03 & 01 \\
\hline \multirow[t]{2}{*}{ cg18608055 } & 19 & SBNO2 & Body & OpenSea & -0.03 & $7.08 \mathrm{E}-$ & $2.42 \mathrm{E}-$ & $5.21 \mathrm{E}-$ & $6.88 \mathrm{E}-$ \\
\hline & & & & & & 09 & 03 & 06 & 01 \\
\hline \multirow[t]{2}{*}{$\operatorname{cg} 16936953$} & 17 & VMP1 & Body & OpenSea & -0.07 & $3.43 \mathrm{E}-$ & $1.17 \mathrm{E}-$ & $2.23 \mathrm{E}-$ & $8.08 \mathrm{E}-$ \\
\hline & & & & & & 11 & 05 & 04 & 01 \\
\hline
\end{tabular}

Footnote: Positive value indicated increased methylation in cases compared to controls, negative values indicated hypomethylation in IBD cases versus controls. NA denotes 
methylation probes with no annotated gene symbol. TSS200: 200bp from transcription start site. Body: within body of the gene

Table 4: Patient demographics for predicting disease course in Inflammatory Bowel Disease 


\begin{tabular}{|c|c|c|}
\hline \multicolumn{3}{|c|}{ Predicting Disease Course in Inflammatory Bowel Disease } \\
\hline Variables & $\begin{array}{l}\text { IBD escalation group } \\
\qquad(n=67)\end{array}$ & $\begin{array}{l}\text { Non-escalation group } \\
\qquad(\mathrm{n}=224)\end{array}$ \\
\hline Subtype IBD(CD:UC:IBD-U) & $39: 26: 2$ & $77: 127: 20$ \\
\hline Edin:Oslo:Orebro:Spain & $28: 21: 14: 4$ & $85: 77: 41: 21$ \\
\hline Males (\%) & $39(58)$ & $118(53)$ \\
\hline $\begin{array}{l}\text { Smoking status (current: never: ex: } \\
\text { missing) }\end{array}$ & $16: 35: 15: 1$ & $37: 104: 82: 1$ \\
\hline Mean age (range) & $32(18-67)$ & $36(18-79)$ \\
\hline \multicolumn{3}{|l|}{ Montreal classification for CD } \\
\hline L1 & 13 & 27 \\
\hline L2 & 9 & 23 \\
\hline L3 & 17 & 26 \\
\hline L4 & 0 & 1 \\
\hline Not available & 0 & 0 \\
\hline \multicolumn{3}{|l|}{ Montreal Behaviour for CD } \\
\hline $\mathrm{B} 1+\mathrm{B} 1 \mathrm{p}$ & 29 & 66 \\
\hline B2 & 6 & 4 \\
\hline B3+B3p & 4 & 5 \\
\hline Not available & 0 & 2 \\
\hline \multicolumn{3}{|l|}{ Paris Extent for UC } \\
\hline E1 & 0 & 41 \\
\hline E2 & 8 & 41 \\
\hline E3 & 18 & 45 \\
\hline Not available & 0 & 0 \\
\hline
\end{tabular}

Table 5: Top 3 CpGs associated with escalation in treatment (surgery or anti-TNF therapies) in Inflammatory Bowel Disease (IBD). HR: hazard ratio; Chr: chromosome 


\begin{tabular}{|c|c|c|c|c|}
\hline CpG probe & Gene & Chr & $\begin{array}{l}\text { IBD HR } \\
\text { Training } \\
\quad \text { Set }\end{array}$ & Function/Relevance in Inflammatory Bowel Disease \\
\hline $\operatorname{cg} 26033526$ & TAP1 & 6 & 12.79 & $\begin{array}{l}\text { MHC-II encoded gene are essential in HLA class I } \\
\text { proteins for generation of cytotoxic T-cell mediated } \\
\text { immune reponse. }\end{array}$ \\
\hline $\operatorname{cg} 20502501$ & RPTOR & 17 & 1.47 & $\begin{array}{l}\text { Encodes an mTOR binding protein and is part of the } \\
\text { mammalian target of rapamycin complex } 1 \\
\text { (mTORC1); mTORC1 plays a role in the } \\
\text { pathogenesis of } \mathrm{UC}^{45} \text {. }\end{array}$ \\
\hline $\operatorname{cg} 06352538$ & TESPA1 & 12 & 1.29 & $\begin{array}{c}\text { Plays a role in development and maturation of T- } \\
\text { cells and T-cell antigen receptor mediated activation } \\
\text { of ERK and NFAT signalling }{ }^{58} \text {. }\end{array}$ \\
\hline
\end{tabular}

Figures 

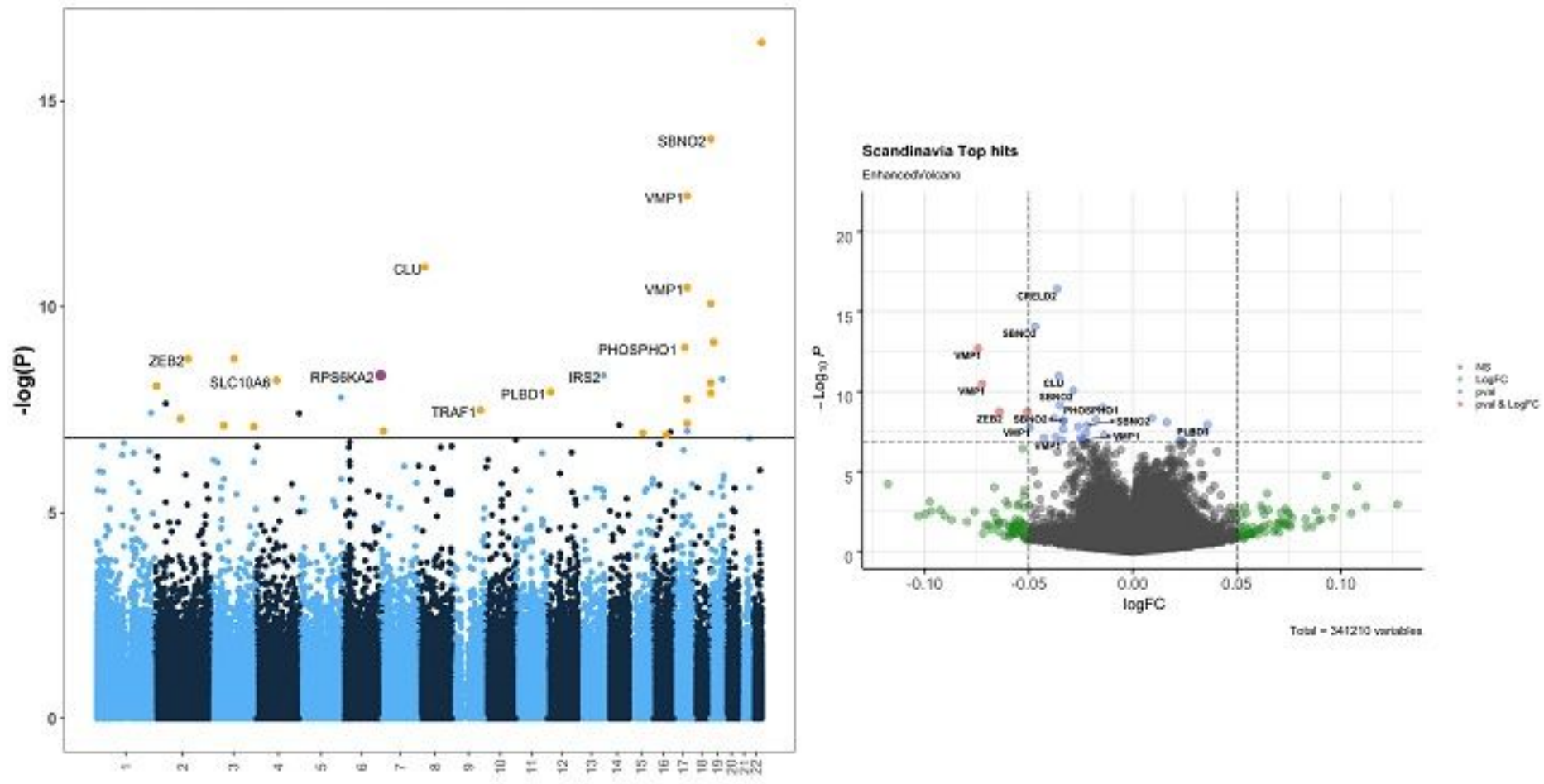

Figure 1

A) Manhattan plot of top differentially methylated positions (DMPs) in IBD versus control in Scandinavia. Yellow dots represent hits that replicate in the UK cohort and purple dot represent hits that replicate in Spain. B) Volcano plot of the top differentially methylated positions (DMPs) across Scandinavia. Horizontal dashed line represents holm significance. Footnote: $Y$-axis for both plots represent $-\log 10(p-$ values) with the horizontal line representing statistical significance after Holm testing. 


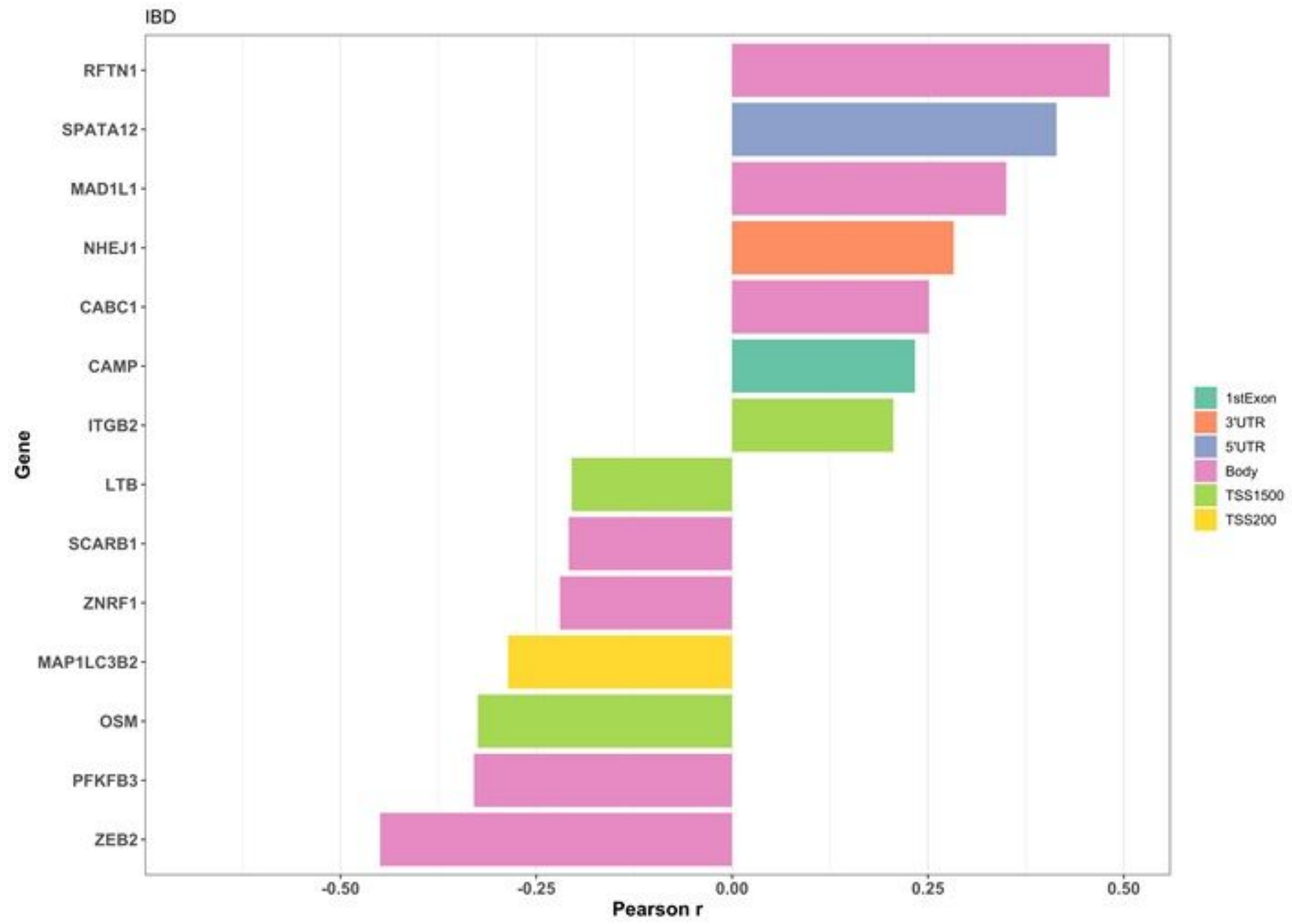

Figure 2

Correlation between DNA methylation probes and gene expression in IBD $(\mathrm{Holm} \mathrm{p}<0.05)$ where no significant correlation was seen in non-IBD for these probes (Holm $p>0.05$ ). Y-axis represents gene names. Bar colours represent CpG probe location within the gene. Footnote: 200 or 1500 nucleotides upstream of the transcriptional start sites (TSS200 and TSS1500). Within regulatory regions of a gene (5'untranslated region (5'UTR), 3' untranslated region ( $\left.3^{\prime} \mathrm{UTR}\right)$ ) or body of the gene (Body). 


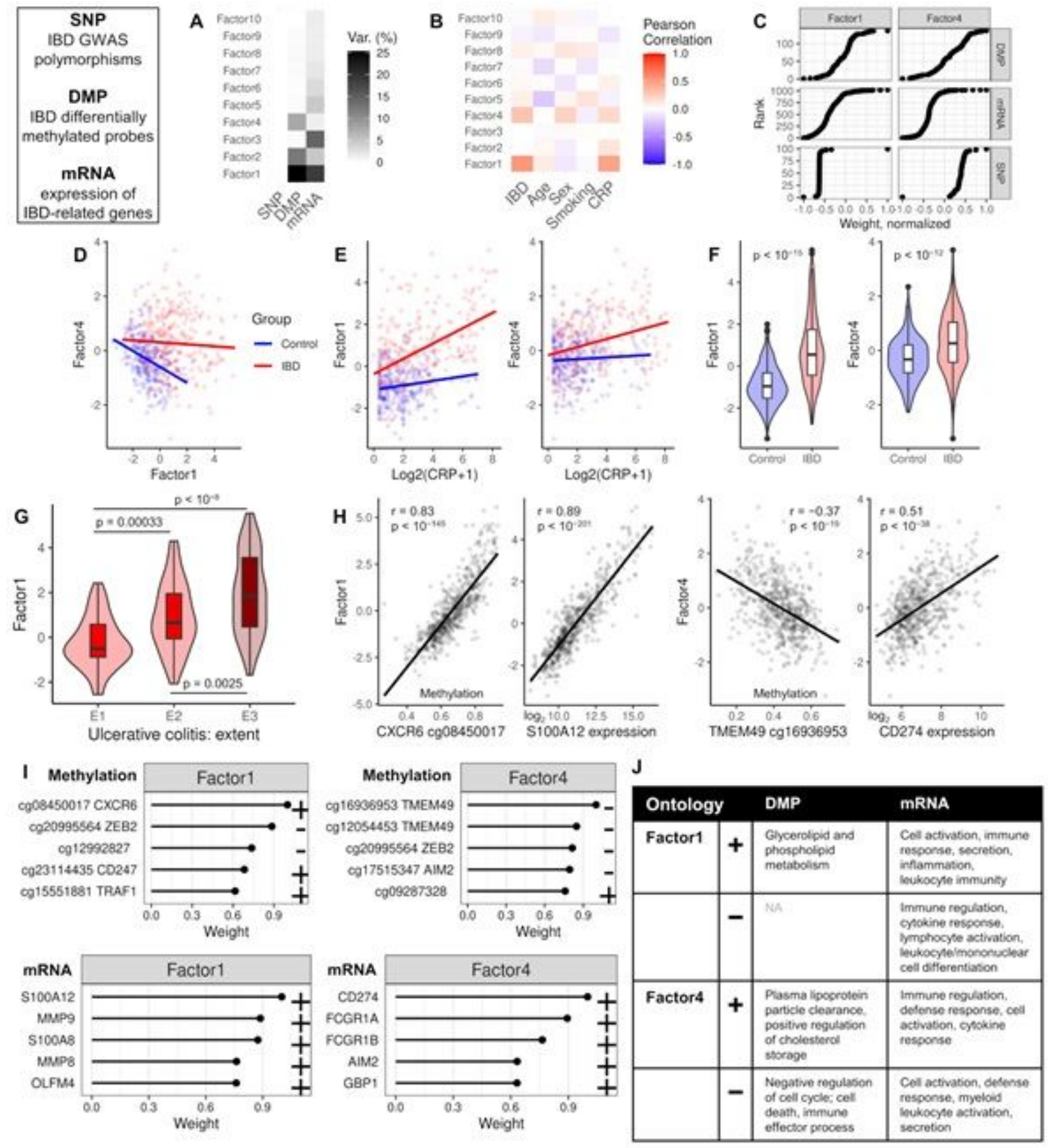

Figure 3

Multi-omic Factor Analysis (MOFA) integrating IBD genome-wide association analysis (GWAS) polymorphisms, mRNA expression of IBD related genes and differentially IBD-related methylated positions (DMPs). A) MOFA heatmap demonstrating the variance explained by each factor. B-E) Analyses demonstrating the correlation of the MOFA factors with inflammatory markers such as hsCRP, IBD, age and smoking. F) Boxplots for Factor 1 and 4 in IBD and controls $\mathrm{G}$ ) Boxplot showing the association of Factor 1 with disease extent in UC. $\mathrm{H}-\mathrm{I}$ ) Correlation plots of the key methylation and mRNA expression genes that define Factor 1 and Factor 4. J) Gene ontology analysis of the top MOFA Factor 1 and 4. 


\section{3-Methylation probe Model}

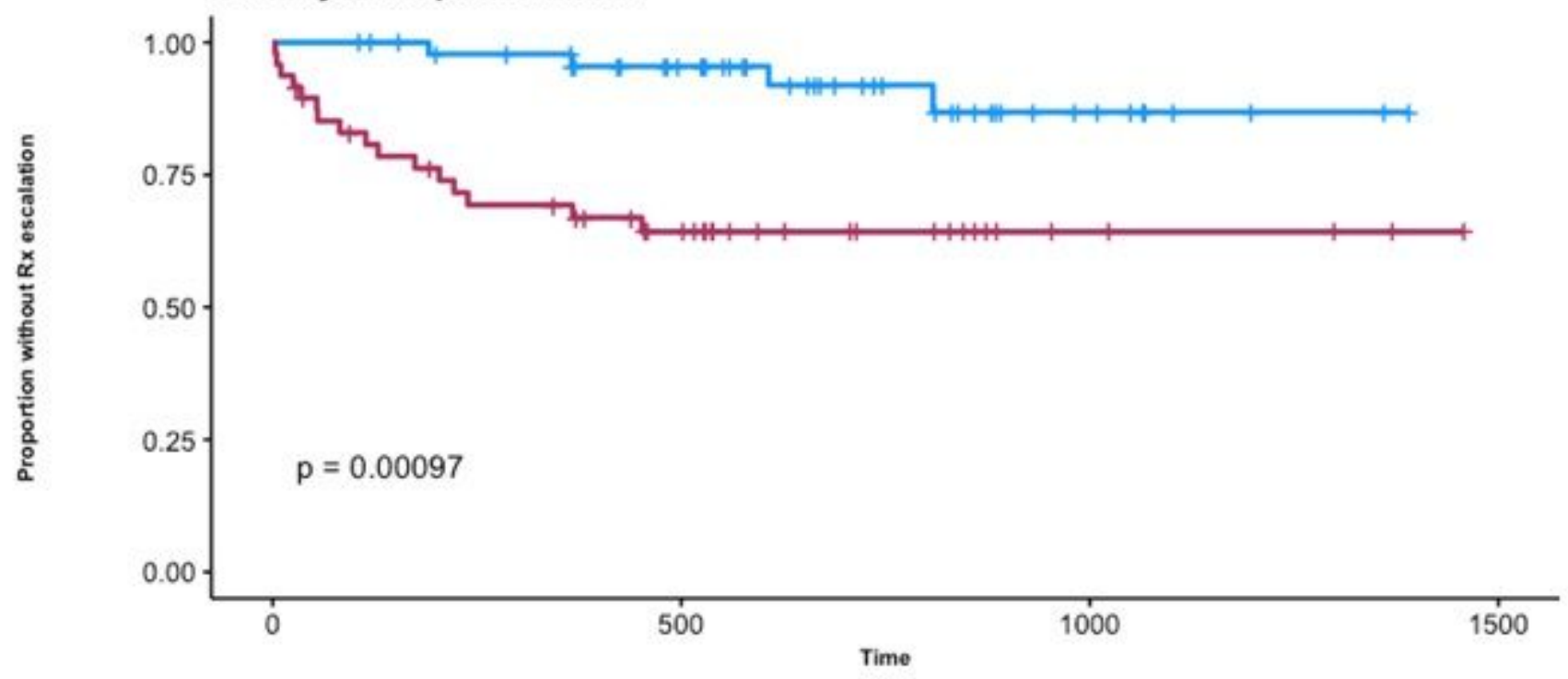

\section{3-Methylation probe Model}

\begin{tabular}{|c|c|c|c|c|}
\hline Low Risk. & 49 & 34 & 8 & 0 \\
\hline High Risk. & 48 & 22 & 4 & 0 \\
\hline & $\dot{0}$ & 500 & 1000 & 1500 \\
\hline
\end{tabular}

\section{Figure 4}

A 3-probe methylation panel predicts treatment escalation in Inflammatory Bowel Disease (IBD). Kaplan meier survival curve demonstrating the proportion of IBD patients who did not require a treatment escalation at diagnosis. High (red) and low (blue) risk represents patient groups defined as high or low risk of treatment escalation based on the 3-probe panel.

\section{Supplementary Files}

This is a list of supplementary files associated with this preprint. Click to download.

- SupplTables.docx

- SupplementaryMaterials.pdf 